\title{
Can Plasmon Change Reaction Path? : Decomposition of Unsymmetrical lodonium Salts as an Organic Probe
}

\section{Miliutina, Elena}

2020-07-16

Miliutina , E , Guselnikova , O , S. Soldatova , N , Bainova , P , Elashnikov , R , Fitl , P , pÿKurten , T , S. Yusubov, M , 'vorík, V , Valiev , R, M. Chehimi , M , Lyutakov, O \& S. Postnikov , P 2020 , ' Can Plasmon Change Reaction Path? Decomposition of Unsymmetrical lodonium Salts as an Organic Probe ' , Journal of Physical Chemistry Letters pÿ, vol. 11 , no. 14 , pp. 57705776 . https://doi.org/10.1021/acs.jpclett.0c01350

http://hdl.handle.net/10138/331978

https://doi.org/10.1021/acs.jpclett.0c01350

acceptedVersion

Downloaded from Helda, University of Helsinki institutional repository.

This is an electronic reprint of the original article.

This reprint may differ from the original in pagination and typographic detail.

Please cite the original version. 


\section{Can Plasmon Change Reaction Path? Decomposition of Unsymmetrical Iodonium Salts as an Organic}

\section{Probe}

Elena Miliutina,${ }^{[a, b]}$ Olga Guselnikova,${ }^{*}[a, b]$ Natalia S. Soldatova,${ }^{[c]}$ Polina Bainova, ${ }^{[b]}$ Roman Elashnikov, ${ }^{[b]}$ Přemysl Fitl, ${ }^{[b]}$ Theo Kurten, ${ }^{[d]}$ Mekhman S. Yusubov, ${ }^{[a]}$ Václav Švorčik, ${ }^{[b]}$ Rashid R. Valiev, ${ }^{[a, d]}$ Mohamed M. Chehimi, ${ }^{[e]}$ Oleksiy Lyutakov ${ }^{[b]}$ and Pavel S. Postnikov ${ }^{*[a, b]}$

[a] Research School of Chemistry \& Applied Biomedical Sciences, National Research Tomsk Polytechnic University, Lenin Avenue 30, Tomsk 634050, Russia, E-mail: postnikov@tpu.ru, guselnikovaoa@tpu.ru

[b] Institute of Chemical Technology, Technicka 5, Prague, 16628 Czech Republic

[c] Institute of Chemistry, Saint Petersburg State University, Universitetskaya Nab., 7/9, Saint Petersburg, Russian Federation

[d] Department of Chemistry, University of Helsinki, Helsinki FIN-00014, Finland

[e] Prof. Mohamed M. Chehimi, University Paris-Est Creteil

Author information

\section{Corresponding Author}

*Pavel S. Postnikov - Research School of Chemistry \& Applied Biomedical Sciences, National Research Tomsk Polytechnic University, Lenin Avenue 30, Tomsk 634050, Russia; Department 
of Solid State Engineering, Institute of Chemical Technology, 16628 Prague, Czech Republic; Email: postnikov@tpu.ru

*Olga Guselnikova - Research School of Chemistry \& Applied Biomedical Sciences, National Research Tomsk Polytechnic University, Lenin Avenue 30, Tomsk 634050, Russia; Department of Solid State Engineering, Institute of Chemical Technology, 16628 Prague, Czech Republic; Email: guselnikovaoa@tpu.ru

\begin{abstract}
Plasmon-assisted transformations of organic compounds represent a novel opportunity for conversion of light to chemical energy at room temperature. However, the mechanistic insights of interaction between plasmon energy and organic molecules is still under debate. Herein, we proposed a comprehensive study of the plasmon-assisted reaction mechanism using unsymmetric iodonium salts (ISs) as an organic probe. The experimental and theoretical analysis allow to exclude the possible thermal effect or hot electron transfer. We found that plasmon interaction with unsymmetrical ISs led to the intramolecular excitation of electron followed by the regioselective cleavage of $\mathrm{C}-\mathrm{I}$ bond with the formation of electron-rich radical species, which cannot be explained by the hot electron excitation or thermal effects. The high regioselectivity is explained by the direct excitation of electron to LUMO with the formation of dissociative excited state according to quantum-chemical modeling, which provide a novel opportunities to the fine control of reactivity using plasmon energy.
\end{abstract}




\section{TOC Graphic}

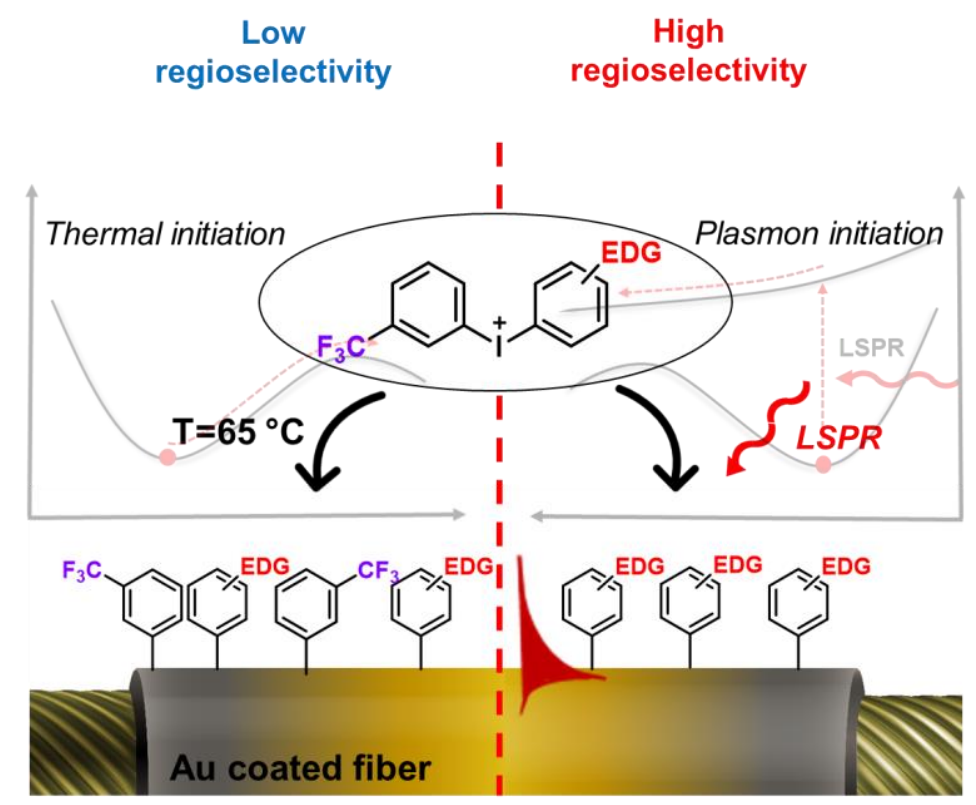

Keywords: iodonium salt, plasmon-assisted reaction, regioselectivity, surface modification, mechanism.

The plasmon can be considered as one the most groundbreaking approach for the activation of organic reactions. ${ }^{1-8}$ Nowadays, plasmon activation has been widely applied for the Pd-catalyzed cross-coupling reactions, ${ }^{9-11}$ hydrogenation, ${ }^{12-15}$ cycloaddition, ${ }^{16,17}$ oxidative reactions, ${ }^{18-20}$ polymerization, ${ }^{21-23}$ methane reforming, ${ }^{24}$ and so forth, whereby the utilization of plasmon allows achieving good yield of desired compounds under extremely mild conditions. Nevertheless, despite these impressive advances, the exact mechanism of plasmon activation of organic compounds has not been fully described yet. ${ }^{3,25,26}$ Hence, a vivid discussion is currently on-going regarding the critical question: how does the energy of plasmon induce the excitation of molecule to the transition state? ${ }^{27-}$ ${ }^{31}$ As a result, three main hypotheses have been proposed: (1) reaction acceleration through 
plasmonic heating, (Figure 1A); ${ }^{32-34}$ (2) transfer of a hot electron to the organic molecule, followed by the formation and relaxation of the excited state (Figure 1B); and (3) intramolecular excitation of an electron to the LUMO orbitals via the decay of the optically excited surface plasmon (SP) (Figure 1B)..$^{28,35,36}$

The all proposed mechanisms have been probed by theoretical and experimental studies in a range of (organic) transformations, but the final point in these discussions have not been set. One possible key issue into understanding the mechanism of plasmon-induced organic reactions involves analyzing their regioselectivity, which may act as a reporter of the reaction path of the interaction of the plasmon with the organic molecule. According to the published results, both the plasmonic heating and the hot electron transfer should lead to the formation of similar products, in contrast to the intramolecular. The modern studies have been mainly dedicated to the chemoselectivity problem, especially in the hydrogenation of acetylene, ${ }^{12,13,15,37}$ oxidation of benzylic alcohol ${ }^{19}$ and reduction of nitrocompounds. ${ }^{38}$ The regioselectivity has been studied poorly, with only a handful of contributions dedicated to these issues, such as the regioselective cleavage of the $\mathrm{C}-\mathrm{O}$ bond in the $\mathrm{S}_{\mathrm{N}} 2$ reaction. ${ }^{36}$ However, the regioselectivity study is able to provide a deeper insight into the mechanism issues from the chemistry point of view and predict new possible pathways for plasmon-induced transformations.

Recently we reported the plasmon-induced modification of thin gold films by symmetric iodonium salts (ISs). ${ }^{39}$ Obviously, in the case of symmetric IS, the regioselectivity of homolytic decomposition is not an issue. However, the application of unsymmetrical ISs for the surface modification deserves more attention due to the lower price and versatile synthetic procedures. ${ }^{40-43}$ In general, the selectivity of C-I bond cleavage, especially 
homolytic, can be considered as the highly challenging issue in the chemistry of ISs. ${ }^{44,45}$ The homolytic decomposition of unsymmetrical ISs often leads to the formation of product mixtures with extremely low selectivity, ${ }^{46-49}$ as seen in the application of unsymmetrical ISs for the modification of carbon surfaces. ${ }^{39,43,50,51}$ Nevertheless, improved selectivity towards the formation of radicals of the electron-rich aryl substituents has been observed in the SET-reactions, such as in the metal-free coupling of ISs. ${ }^{52-54}$ Thus, the evaluation of regioselectivity issues has a great demand from the plasmon-assisted reactions mechanisms as well as from the common reactivity point of view.

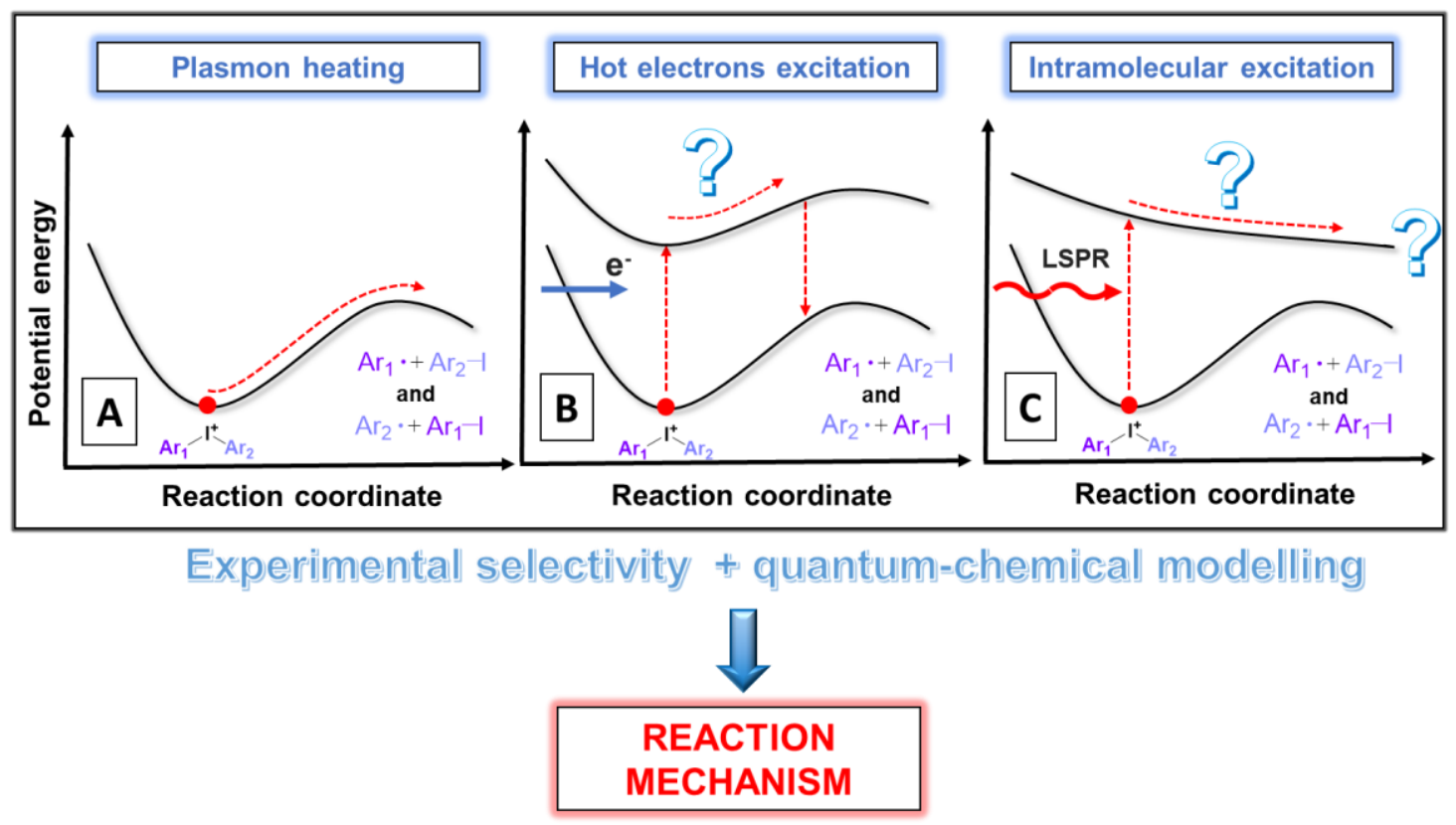

Figure 1. Proposed mechanisms for plasmon-assisted reactions: (A) plasmonic heating; (B) transfer of hot carrier to the organic molecule followed by the formation and relaxation of excited state; (C) intramolecular excitation of electron.

In this contribution, we perform in-depth study of plasmon-driven regioselective homolysis of $\mathrm{C}-\mathrm{I}$ bond in the unsymmetrical ISs using the surface modification of gold-coated optic fibers 
and quantum-chemical calculations (Scheme 1). Moreover, we demonstrate the extraordinary regioselectivity of C-I bond homolysis under plasmon excitation leading to the selective transfer of the more electron-rich aryl radical to the surface, which opens up new opportunities for the design of functional materials.

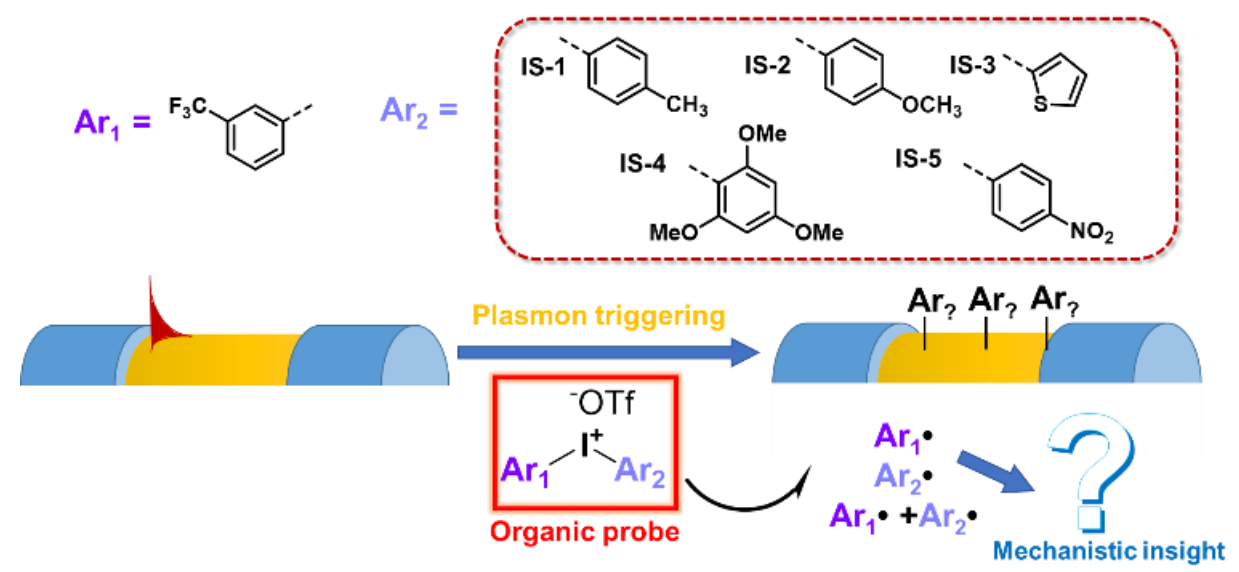

Scheme 1 Utilization of unsymmetrical ISs $\mathbf{1}-\mathbf{5}$ as an organic probe for plasmon-induced reactions.

In order to study the reactivity of the ISs, the series of salts 1-5 was prepared containing the electron-poor 3-(trifluoromethyl)phenyl and the electron-rich moieties (Scheme 1, 1-4) and (4nitrophenyl)(3-(trifluoromethyl)phenyl)iodonium triflate 5 containing two electron-withdrawing substituents according to published procedure. ${ }^{55-57}$ These precursors were then subjected by the plasmon-assisted homolyses using LED irradiation with $\lambda=595 \mathrm{~nm}$ (corresponded to the maximum of the plasmon resonance peak of fiber - Figure S1D) using the gold-coated optical fiber, where the plasmon has been excited by the light passing through the fiber in order to prevent the direct interaction of ISs with photons (Scheme 1). ${ }^{39,58}$ The gold-coated fibers were immersed in the solution of ISs 1-5 (3 mM in water/methanol (2:1)) and the surface modification progress was 
monitored by the shift of the SPR maximum (Figure S3-S7). It should be also noted that the reaction with ISs without light did not lead to the formation of an organic layers on the surface (SI) (Figure S2), which is in a good agreement with previously published data. ${ }^{39}$ Similarly, LED illumination/irradiation at $\lambda=405 \mathrm{~nm}$ did not result in any changes in surface properties (Figure S9). So, reaction take place only under plasmon activation of ISs. In the next step we performed the estimation of the surface grafting time using the saturation of plasmon absorption band shift for different IS as a function of substituent. Surprisingly, the time required for the full saturation of available surface sites for the modification was found to be independent of the type of ISs. Thus, full coverage was completed after 120 minutes (saturation of the SPR shift) with the formation of homogenous organic close-to-monomolecular layer (according to AFM measurements, Figure S8).

The chemical composition of layers has been studied by SEM-EDX, XPS, Raman spectroscopy and AFM (detailed assignation is provided in Figure S1-S9). According to the common views on the thermal mechanism (Figure 1A), we expected the formation of mixed layers containing both aryl groups - electron-rich and electron poor as in conventional heating. ${ }^{40,50}$ Surprisingly, for the ISs 1-4, we did not observe the presence of the $\mathrm{CF}_{3}$ groups in the XPS and Raman spectra (Figure S3-S8). On the Raman spectra of gold fiber modified by ISs $\mathbf{1}-\mathbf{4}$ we did not observe high intensity peaks in the region $1100-1300 \mathrm{~cm}^{-1}$, indicating the absence of $\mathrm{CF}_{3}$ groups (Table S1). However, we found Raman peaks, which can be assigned to the electron-donating part of ISs (Figure S3AS7A, Table S1). For instance, on the Raman spectra of fiber with grafted IS-1, there are characteristic vibrations of methylphenyl groups at: 816, 968, $1467 \mathrm{~cm}^{-1}\left(\mathrm{Ar}_{\mathrm{C}-\mathrm{H}}\right), 1564,1431,504$, 586, $484\left(\mathrm{Ar}_{\mathrm{C}-\mathrm{C}}\right)$. 
The observed effects are able to sufficiently improve the applicability of ISs for the modification and introduction of reactive groups to the surface because the introduction of reactive amino-groups have not been possible via iodonium chemistry due to unavailability of substrates. Such unusual reactivity of ISs can be explained by the effects of plasmon on the reaction path. Mentioned above concepts about plasmon interaction with organic molecules consume the initial excitation of electrons to LUMO orbital (intrinsic (Figure 1C) or hot electrons (Figure 1B)) or the simple plasmonic heating (Figure1A).

In order to exclude the heating effects on the $\mathrm{C}-\mathrm{I}$ bond homolysis, we performed a range of control experiments. Firstly, for the rough estimation of reaction temperature, we tracked the temperature of gold-coated fiber during the plasmon-induced grafting of IS-1. As have been reported previously, the plasmon excitation is able to promote the local heating, which can affect on the reaction pathway. ${ }^{59}$ Thermovision measurements revealed the increase of the fiber temperature by $\sim 1{ }^{\circ} \mathrm{C}$ after 2 hours of irradiation (Figure S10 A,B), ruling out the possible thermal IS-1 decomposition during irradiation of the sample during plasmon excitation (see Fig. S10 and related discussion). An additional control involved the simple heating of IS-1 solution in the presence of the plasmonic fiber at $40{ }^{\circ} \mathrm{C}$ (i.e. some $10^{\circ} \mathrm{C}$ above the temperature reached during the irradiation) for 3 hours, without any noticeable changes in XPS and Raman spectra. Only increase of temperature to $60^{\circ} \mathrm{C}$ resulted in spectral changes: the XPS analysis as well as Raman spectroscopy revealed the formation of mixed layers containing close-to-equal content of (3(trifluoromethyl)phenyl and 4-methylphenyl) groups (Figure S10). Thus, the simple plasmon heating cannot explain the regioselectivity of $\mathrm{C}-\mathrm{I}$ bond homolysis. Due to this reason, we applied the quantum-chemical modelling for the explanation of the observed regioselectivity. 
In contrast, the homolysis of the IS-5 under plasmon led to the formation of mixed layers containing both the $3-\mathrm{CF}_{3}$ - and $4-\mathrm{NO}_{2}$-groups (Figure 2, Figure $\mathrm{S} 7$ ). This fact was confirmed by the appearance of strong Raman peaks of nitro group at 1312 and $768 \mathrm{~cm}^{-1}$ and $\mathrm{CF}_{3}$ groups -1081 , 1150, $1265 \mathrm{~cm}^{-1}$. Moreover, we observed the partial reduction of the $\mathrm{NO}_{2}$ groups to the $\mathrm{NH}_{2}$ moieties, presumably via the well-known plasmon-assisted process (appearance of peaks at 1050 and $1592 \mathrm{~cm}^{-1}$ and N-H component of N1s XPS signal at $\left.400 \mathrm{eV}\right)^{60}$.
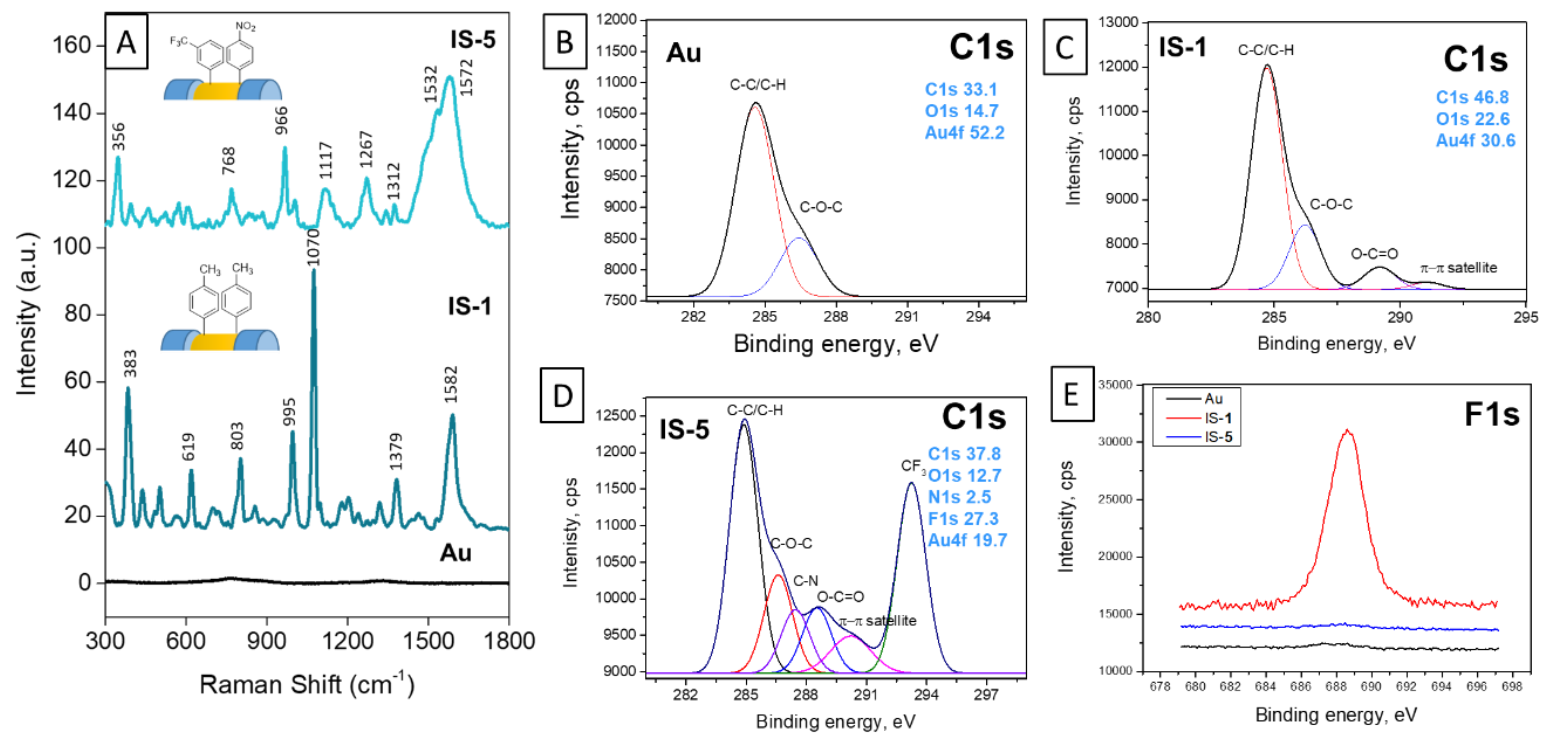

Figure 2. (A) SERS spectra of gold-coated fiber before and after decomposition and grafting of IS-1, IS-5 after irradiation; XPS spectra: C1 region of (B) pristine gold fiber, (C) decomposition of IS-1 after irradiation, (D) decomposition of IS-5 after irradiation, (E) F1s region of gold fiber before and after decomposition of IS-1, IS-5 after irradiation.

Initially, we carried out the modelling of three possible pathways for the homolysis of C-I bonds in the ISs 1-5. As expected, the enthalpy of activation for the homolysis of both C-I bonds are similar (see section 9.1 in SI). Thus, we analyzed the effect of plasmon on the organic molecule 
in frame of the aforementioned hypothesizes. First model includes the excitation of hot electron to the ISs with the formation of neutral radical specie in $\mathrm{D}_{1}$ state $(143 \mathrm{kcal} / \mathrm{mol})$ (Figure $\left.3 \mathrm{~A}\right)$. The relaxation of $\mathrm{D}_{1}$ state led to the formation of dissociative $\mathrm{D}_{0}$ state $(3.9 \mathrm{kcal} / \mathrm{mol})$, which spontaneously decomposed to the appropriate 3-(trifluoromethyl)phenyl radical and 4-iodotoluene (see section 9.2 in SI). Thus, according to the calculation results, the reaction pathway based on the excitation of hot electrons and the formation of neutral radical did not correlate with the observed reaction regioselectivity.

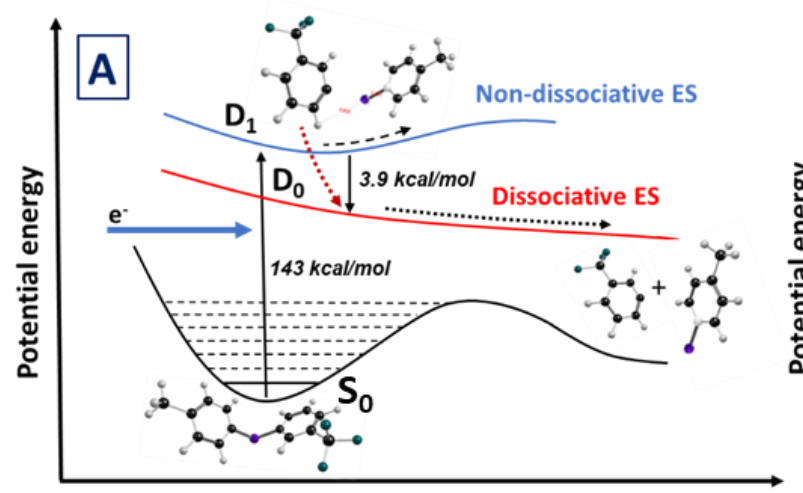

Reaction coordinate
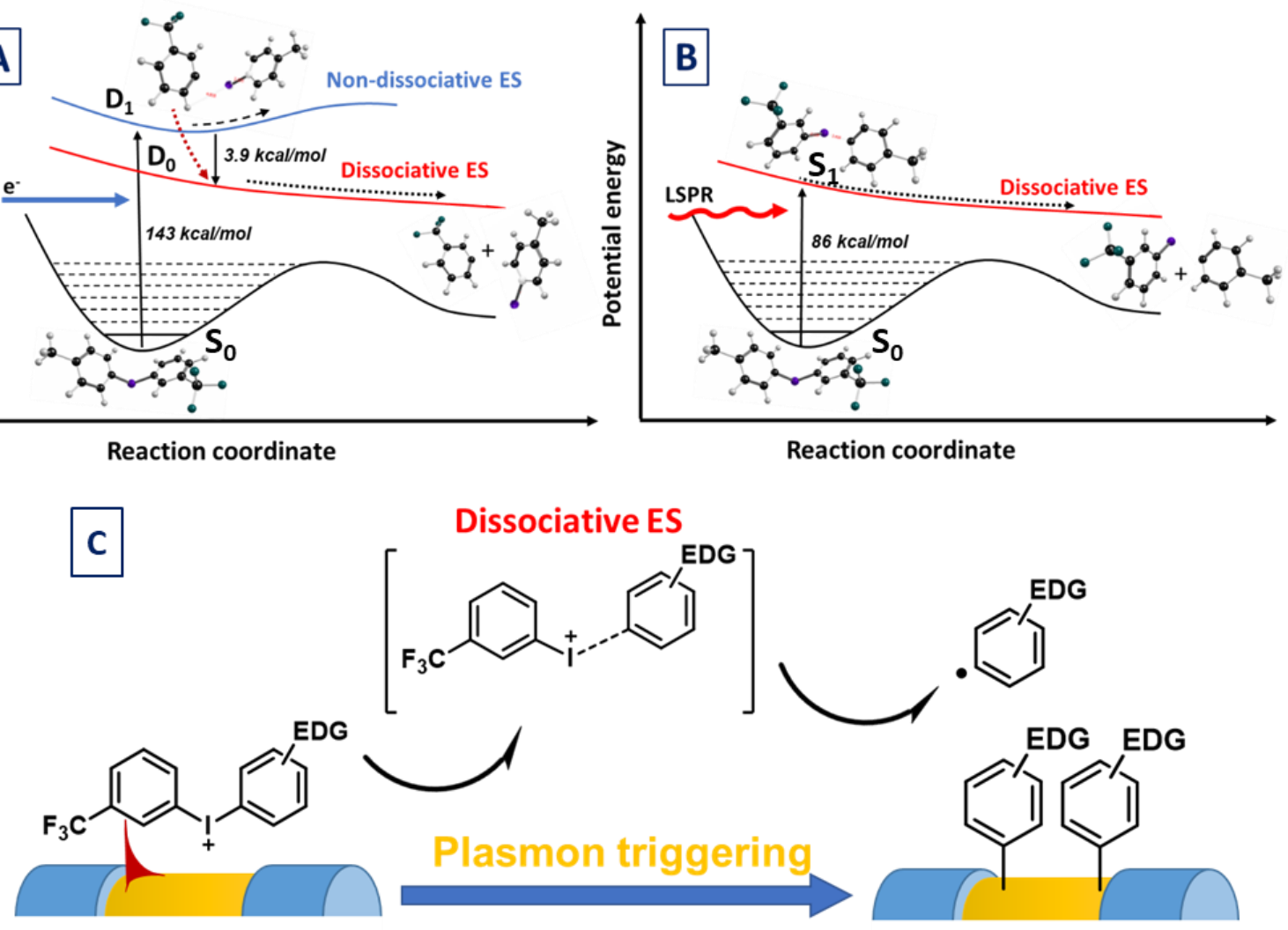

Figure 3. (A, B) Quantum-chemical evaluation of plasmon-assisted homolysis of C-I bonds in IS-1. (C) Plausible mechanism. 
Next, we carried out the TDDFT-calculation of the process depicted in Figure 3B involving the intramolecular excitation of an electron from LUMO to HOMO through the decay of SP, as has been proved by Kim. ${ }^{28}$ The excitation of electron led to the formation of positively charged excited state $S_{1}$, which has been found to be dissociative (according to Figure 3). Moreover, the cleavage of C-I bond in this case proceeded with the homolysis of C-I bond located on electron-rich aryl groups $(86 \mathrm{kcal} / \mathrm{mol})$. This tendency has been observed in all range of ISs bearing electrondonating aryl groups (see section 9.3 in SI and related discussion). Contrariwise, the decomposition of iodonium cation $\mathbf{5}$ led to the formation of both radicals (3(trifluoromethyl)phenyl and 4-nitrophenyl) with perfect fit with experimental data, where the formation of mixed layer have been observed (Figure 2). Noteworthy, that decomposition of IS-5 via excitation by hot electrons led to the formation of 3-(trifluoromethyl)phenyl radical with high regioselectivity, which does not fit to the experimental data.

Thus, the results of theoretical and experimental investigations proved that the plasmon is able to change the reaction pathway and regioselectivity of C-I bond cleavage in the unsymmetrical ISs. Moreover, the proposed reaction can be considered as a one more example of selective organic transformations proceeding through the direct excitation of electron from HOMO to LUMO with the formation of dissociative excited state. ${ }^{61,62}$ We believe, that the observed effects are able to sufficiently explore the border of plasmon application in organic synthesis and provide the deeper insight to the mechanism of interaction between plasmon and organic compounds.

Associated content

\section{Supporting Information.}


The following files are available free of charge.

Characterization of pristine gold-coated fiber (Figure S1). Control experiment without light (Figure S2). Plasmon induced grafting of ISs 1-5 (Figure S3-S7). Assignation of SERS peaks. Characterization of surface by AFM analysis (Figure S8). Control experiment with alternative wavelength (Figure S9). Control experiment with thermal induced grafting of IS-1 (Figure S10). Description of the samples preparation and measurement techniques. Description of the procedure for obtaining of ISs 1-5. Calculation details and geometry of ISs 1-5 ( $\left.\mathrm{S}_{1}, \mathrm{~S}_{2}, \mathrm{D}_{0}\right)$ and decomposition pathways. Thermal decomposition of IS-1.

Acknowledgment

This work was supported by the Russian Science Foundation (RSF-17-73-20066) and by GACR under projects 19-24603 Y. PSP acknowledges Dr. A. Shafir (IQAC-CSIC) for the fruitful discussions.

\section{References}

(1) Zhang, N.; Han, C.; Fu, X.; Xu, Y.-J. Function-Oriented Engineering of Metal-Based Nanohybrids for Photoredox Catalysis: Exerting Plasmonic Effect and Beyond. Chem 2018, 4 (8), $1832-1861$.

(2) Choi, C. H.; Chung, K.; Nguyen, T.-T. H.; Kim, D. H. Plasmon-Mediated Electrocatalysis for Sustainable Energy: From Electrochemical Conversion of Different Feedstocks to Fuel Cell Reactions. ACS Energy Lett. 2018, 3 (6), 1415-1433.

(3) Zhang, X.; Ke, X.; Yao, J. Recent Development of Plasmon-Mediated Photocatalysts and Their Potential in Selectivity Regulation. J. Mater. Chem. A 2018, 6 (5), 1941-1966. 
(4) Zrimsek, A. B.; Chiang, N.; Mattei, M.; Zaleski, S.; McAnally, M. O.; Chapman, C. T.; Henry, A.-I.; Schatz, G. C.; Van Duyne, R. P. Single-Molecule Chemistry with Surface- and Tip-Enhanced Raman Spectroscopy. Chem. Rev. 2017, 117 (11), 7583-7613.

(5) Meng, X.; Liu, L.; Ouyang, S.; Xu, H.; Wang, D.; Zhao, N.; Ye, J. Nanometals for Solar-toChemical Energy Conversion: From Semiconductor-Based Photocatalysis to Plasmon-Mediated Photocatalysis and Photo-Thermocatalysis. Adv. Mater. 2016, 28 (32), 6781-6803.

(6) Linic, S.; Aslam, U.; Boerigter, C.; Morabito, M. Photochemical Transformations on Plasmonic Metal Nanoparticles. Nat. Mater. 2015, 14 (6), 567-576.

(7) Zhan, C.; Chen, X.-J.; Yi, J.; Li, J.-F.; Wu, D.-Y.; Tian, Z.-Q. From Plasmon-Enhanced Molecular Spectroscopy to Plasmon-Mediated Chemical Reactions. Nat. Rev. Chem. 2018, 2 (9), 216-230.

(8) Shin, H.-H.; Koo, J.-J.; Lee, K. S.; Kim, Z. H. Chemical Reactions Driven by Plasmon-Induced Hot Carriers. Appl. Mater. Today 2019, 16, 112-119.

(9) Wang, F.; Li, C.; Chen, H.; Jiang, R.; Sun, L.-D.; Li, Q.; Wang, J.; Yu, J. C.; Yan, C.-H. Plasmonic Harvesting of Light Energy for Suzuki Coupling Reactions. J. Am. Chem. Soc. 2013, 135 (15), 5588-5601.

(10) Xiao, Q.; Sarina, S.; Bo, A.; Jia, J.; Liu, H.; Arnold, D. P.; Huang, Y.; Wu, H.; Zhu, H. Visible Light-Driven Cross-Coupling Reactions at Lower Temperatures Using a Photocatalyst of Palladium and Gold Alloy Nanoparticles. ACS Catal. 2014, 4 (6), 1725-1734. 
(11) Verma, P.; Kuwahara, Y.; Mori, K.; Yamashita, H. Pd/Ag and Pd/Au Bimetallic Nanocatalysts on Mesoporous Silica for Plasmon-Mediated Enhanced Catalytic Activity under Visible Light Irradiation. J. Mater. Chem. A 2016, 4 (26), 10142-10150.

(12) Guselnikova, O.; Olshtrem, A.; Kalachyova, Y.; Panov, I.; Postnikov, P.; Svorcik, V.; Lyutakov, O. Plasmon Catalysis on Bimetallic Surface-Selective Hydrogenation of Alkynes to Alkanes or Alkenes. J. Phys. Chem. C 2018, 122 (46), 26613-26622.

(13) Landry, M. J.; Gellé, A.; Meng, B. Y.; Barrett, C. J.; Moores, A. Surface-Plasmon-Mediated Hydrogenation of Carbonyls Catalyzed by Silver Nanocubes under Visible Light. ACS Catal. 2017, 7 (9), 6128-6133.

(14) Yin, Z.; Wang, Y.; Song, C.; Zheng, L.; Ma, N.; Liu, X.; Li, S.; Lin, L.; Li, M.; Xu, Y.; et al. Hybrid $\mathrm{Au}-\mathrm{Ag}$ Nanostructures for Enhanced Plasmon-Driven Catalytic Selective Hydrogenation through Visible Light Irradiation and Surface-Enhanced Raman Scattering. J. Am. Chem. Soc. 2018, 140 (3), 864-867.

(15) Zhang, X.; Li, X.; Zhang, D.; Su, N. Q.; Yang, W.; Everitt, H. O.; Liu, J. Product Selectivity in Plasmonic Photocatalysis for Carbon Dioxide Hydrogenation. Nat. Commun. 2017, 8 (1), 1-9.

(16) Chuang, C.-C.; Chu, H.-C.; Huang, S.-B.; Chang, W.-S.; Tuan, H.-Y. Laser-Induced Plasmonic Heating in Copper Nanowire Fabric as a Photothermal Catalytic Reactor. Chem. Eng. J. 2020, 379, 122285.

(17) Guselnikova, O.; Postnikov, P.; Chehimi, M. M.; Kalachyovaa, Y.; Svorcik, V.; Lyutakov, O. Surface Plasmon-Polariton: A Novel Way To Initiate Azide-Alkyne Cycloaddition. Langmuir 2019, 35 (6), 2023-2032. 
(18) Xiao, Q.; Connell, T. U.; Cadusch, J. J.; Roberts, A.; Chesman, A. S. R.; Gómez, D. E. HotCarrier Organic Synthesis via the Near-Perfect Absorption of Light. ACS Catal. 2018, 8 (11), $10331-10339$.

(19) Li, H.; Qin, F.; Yang, Z.; Cui, X.; Wang, J.; Zhang, L. New Reaction Pathway Induced by Plasmon for Selective Benzyl Alcohol Oxidation on BiOCl Possessing Oxygen Vacancies. J. Am. Chem. Soc. 2017, 139 (9), 3513-3521.

(20) Zheng, Z.; Tachikawa, T.; Majima, T. Single-Particle Study of Pt-Modified Au Nanorods for Plasmon-Enhanced Hydrogen Generation in Visible to Near-Infrared Region. J. Am. Chem. Soc. 2014, 136 (19), 6870-6873.

(21) Erzina, M.; Guselnikova, O.; Postnikov, P.; Elashnikov, R.; Kolska, Z.; Miliutina, E.; Švorčík, V.; Lyutakov, O. Plasmon-Polariton Induced, "from Surface” RAFT Polymerization, as a Way toward Creation of Grafted Polymer Films with Thickness Precisely Controlled by Self-Limiting Mechanism. Advanced Materials Interfaces 2018, 5 (22), 1801042.

(22) Guselnikova, O.; Marque, S. R. A.; Tretyakov, E. V.; Mares, D.; Jerabek, V.; Audran, G.; Joly, J.-P.; Trusova, M.; Svorcik, V.; Lyutakov, O.; et al. Unprecedented Plasmon-Induced NitroxideMediated Polymerization (PI-NMP): A Method for Preparation of Functional Surfaces. J. Mater. Chem. A 2019, 7 (20), 12414-12419.

(23) Ding, T.; Mertens, J.; Lombardi, A.; Scherman, O. A.; Baumberg, J. J. Light-Directed Tuning of Plasmon Resonances via Plasmon-Induced Polymerization Using Hot Electrons. ACS Photonics 2017, 4 (6), 1453-1458. 
(24) Zhou, L.; Martirez, J. M. P.; Finzel, J.; Zhang, C.; Swearer, D. F.; Tian, S.; Robatjazi, H.; Lou, M.; Dong, L.; Henderson, L.; et al. Light-Driven Methane Dry Reforming with Single Atomic Site Antenna-Reactor Plasmonic Photocatalysts. Nat. Energy 2020, 5 (1), 61-70.

(25) Kazuma, E.; Kim, Y. Mechanistic Studies of Plasmon Chemistry on Metal Catalysts. Angew. Chem.-Int. Edit. 2019, 58 (15), 4800-4808.

(26) Baumberg, J. J. Hot Electron Science in Plasmonics and Catalysis: What We Argue About. Faraday Discuss. 2019, 214 (0), 501-511.

(27) Rao, V. G.; Aslam, U.; Linic, S. Chemical Requirement for Extracting Energetic Charge Carriers from Plasmonic Metal Nanoparticles to Perform Electron-Transfer Reactions. J. Am. Chem. Soc. 2019, 141 (1), 643-647.

(28) Kazuma, E.; Jung, J.; Ueba, H.; Trenary, M.; Kim, Y. Real-Space and Real-Time Observation of a Plasmon-Induced Chemical Reaction of a Single Molecule. Science 2018, 360 (6388), 521526.

(29) Seemala, B.; Therrien, A. J.; Lou, M.; Li, K.; Finzel, J. P.; Qi, J.; Nordlander, P.; Christopher, P. Plasmon-Mediated Catalytic O2 Dissociation on Ag Nanostructures: Hot Electrons or Near Fields? ACS Energy Lett. 2019, 4 (8), 1803-1809.

(30) Jain, P. K. Taking the Heat Off of Plasmonic Chemistry. J. Phys. Chem. C 2019, 123 (40), 24347-24351.

(31) Zhan, C.; Liu, B.-W.; Huang, Y.-F.; Hu, S.; Ren, B.; Moskovits, M.; Tian, Z.-Q. Disentangling Charge Carrier from Photothermal Effects in Plasmonic Metal Nanostructures. Nat. Commun. 2019, $10(1), 1-8$. 
(32) Sivan, Y.; Un, I. W.; Dubi, Y. Assistance of Metal Nanoparticles in Photocatalysis - Nothing More than a Classical Heat Source. Faraday Discuss. 2019, 214 (0), 215-233.

(33) Dubi, Y.; Sivan, Y. "Hot" Electrons in Metallic Nanostructures-Non-Thermal Carriers or Heating? Light-Sci. Appl. 2019, 8 (1), 1-8.

(34) Sarhan, R. M.; Koopman, W.; Schuetz, R.; Schmid, T.; Liebig, F.; Koetz, J.; Bargheer, M. The Importance of Plasmonic Heating for the Plasmon-Driven Photodimerization of 4Nitrothiophenol. Sci Rep 2019, 9 (1), 1-8.

(35) Tesema, T. E.; Kafle, B.; Habteyes, T. G. Plasmon-Driven Reaction Mechanisms: Hot Electron Transfer versus Plasmon-Pumped Adsorbate Excitation. J. Phys. Chem. C 2019, 123 (14), 8469-8483.

(36) Mette, G.; Adamkiewicz, A.; Reutzel, M.; Koert, U.; Dürr, M.; Höfer, U. Controlling an SN2 Reaction by Electronic and Vibrational Excitation: Tip-Induced Ether Cleavage on Si(001). Angew. Chem.-Int. Edit. 2019, 58 (11), 3417-3420.

(37) Quiroz, J.; Barbosa, E. C. M.; Araujo, T. P.; Fiorio, J. L.; Wang, Y.-C.; Zou, Y.-C.; Mou, T.; Alves, T. V.; de Oliveira, D. C.; Wang, B.; et al. Controlling Reaction Selectivity over Hybrid Plasmonic Nanocatalysts. Nano Lett. 2018, 18 (11), 7289-7297.

(38) Cui, L.; Wang, P.; Li, Y.; Sun, M. Selective Plasmon-Driven Catalysis for Para-Nitroaniline in Aqueous Environments. Sci Rep 2016, 6 (1), 1-9.

(39) Miliutina, E.; Guselnikova, O.; Bainova, P.; Kalachyova, Y.; Elashnikov, R.; Yusubov, M. S.; Zhdankin, V. V.; Postnikov, P.; Švorčík, V.; Lyutakov, O. Plasmon-Assisted Activation and 
Grafting by Iodonium Salt: Functionalization of Optical Fiber Surface. Adv. Mater. Interfaces 2018, $5(20), 1800725$.

(40) He, M.; Swager, T. M. Covalent Functionalization of Carbon Nanomaterials with Iodonium Salts. Chem. Mater. 2016, 28 (23), 8542-8549.

(41) Fontanesi, C.; Bortolotti, C. A.; Vanossi, D.; Marcaccio, M. Dissociation Dynamics of Asymmetric Alkynyl(Aryl)Iodonium Radicals: An Ab Initio DRC Approach to Predict the Surface Functionalization Selectivity. J. Phys. Chem. A 2011, 115 (42), 11715-11722.

(42) Stevenson, K. J.; Veneman, P. A.; Gearba, R. I.; Mueller, K. M.; Holliday, B. J.; Ohta, T.; Chan, C. K. Controlled Covalent Modification of Epitaxial Single Layer Graphene on 6H-SiC (0001) with Aryliodonium Salts Using Electrochemical Methods. Faraday Discuss. 2014, 172 (0), 273-291.

(43) Médard, J.; Combellas, C.; Kanoufi, F.; Pinson, J.; Chauvin, J.; Deronzier, A. Patterning Surfaces through Photografting of Iodonium Salts. J. Phys. Chem. C 2018, 122 (34), 19722-19730.

(44) Wang, X.; Studer, A. Iodine(III) Reagents in Radical Chemistry. Acc. Chem. Res. 2017, 50 (7), 1712-1724.

(45) Yoshimura, A.; Zhdankin, V. V. Advances in Synthetic Applications of Hypervalent Iodine Compounds. Chem. Rev. 2016, 116 (5), 3328-3435.

(46) Hartmann, M.; Li, Y.; Mück-Lichtenfeld, C.; Studer, A. Generation of Aryl Radicals through Reduction of Hypervalent Iodine(III) Compounds with TEMPONa: Radical Alkene Oxyarylation. Chem.-Eur. J. 2016, 22 (10), 3485-3490. 
(47) Wen, J.; Zhang, R.-Y.; Chen, S.-Y.; Zhang, J.; Yu, X.-Q. Direct Arylation of Arene and NHeteroarenes with Diaryliodonium Salts without the Use of Transition Metal Catalyst. J. Org. Chem. 2012, 77 (1), 766-771.

(48) Baralle, A.; Fensterbank, L.; Goddard, J.-P.; Ollivier, C. Aryl Radical Formation by Copper(I) Photocatalyzed Reduction of Diaryliodonium Salts: NMR Evidence for a CuII/CuI Mechanism. Chem.-Eur. J. 2013, 19 (33), 10809-10813.

(49) Neufeldt, S. R.; Sanford, M. S. Combining Transition Metal Catalysis with Radical Chemistry: Dramatic Acceleration of Palladium-Catalyzed CH Arylation with Diaryliodonium Salts. Adv. Synth. Catal. 2012, 354 (18), 3517-3522.

(50) Vase, K. H.; Holm, A. H.; Norrman, K.; Pedersen, S. U.; Daasbjerg, K. Covalent Grafting of Glassy Carbon Electrodes with Diaryliodonium Salts: New Aspects. Langmuir 2007, 23 (7), 3786-3793.

(51) Dirk, S. M.; Pylypenko, S.; Howell, S. W.; Fulghum, J. E.; Wheeler, D. R. Potential-Directed Assembly of Aryl Iodonium Salts onto Silicon $\{100\}$ Hydride Terminated and Platinum Surfaces. Langmuir 2005, 21 (24), 10899-10901.

(52) Yamaoka, N.; Sumida, K.; Itani, I.; Kubo, H.; Ohnishi, Y.; Sekiguchi, S.; Dohi, T.; Kita, Y. Single-Electron-Transfer (SET)-Induced Oxidative Biaryl Coupling by PolyalkoxybenzeneDerived Diaryliodonium(III) Salts. Chem.-Eur. J. 2013, 19 (44), 15004-15011.

(53) Morimoto, K.; Nakamura, A.; Dohi, T.; Kita, Y. Metal-Free Oxidative Cross-Coupling Reaction of Thiophene Iodonium Salts with Pyrroles. Eur. J. Org. Chem. 2016, 2016 (25), 42944297. 
(54) Morimoto, K.; Ohnishi, Y.; Koseki, D.; Nakamura, A.; Dohi, T.; Kita, Y. Stabilized Pyrrolyl Iodonium Salts and Metal-Free Oxidative Cross-Coupling. Org. Biomol. Chem. 2016, 14 (38), $8947-8951$.

(55) Soldatova, N.; Postnikov, P.; Kukurina, O.; Zhdankin, V. V.; Yoshimura, A.; Wirth, T.; Yusubov, M. S. One-Pot Synthesis of Diaryliodonium Salts from Arenes and Aryl Iodides with Oxone-Sulfuric Acid. Beilstein J. Org. Chem. 2018, 14 (1), 849-855.

(56) Soldatova, N.; Postnikov, P.; Kukurina, O.; Zhdankin, V. V.; Yoshimura, A.; Wirth, T.; Yusubov, M. S. Facile One-Pot Synthesis of Diaryliodonium Salts from Arenes and Aryl Iodides with Oxone. ChemistryOpen 2017, 6 (1), 18-20.

(57) Seidl, T. L.; Sundalam, S. K.; McCullough, B.; Stuart, D. R. Unsymmetrical Aryl(2,4,6Trimethoxyphenyl)Iodonium Salts: One-Pot Synthesis, Scope, Stability, and Synthetic Studies. $J$. Org. Chem. 2016, 81 (5), 1998-2009.

(58) Guselnikova, O.; Miliutina, E.; Elashnikov, R.; Burtsev, V.; Chehimi, M. M.; Svorcik, V.; Yusubov, M.; Lyutakov, O.; Postnikov, P. Chemical Modification of Gold Surface via UVGenerated Aryl Radicals Derived 3,5-Bis(Trifluoromethyl)Phenyl)Iodonium Salt. Prog. Org. Coat. 2019, 136, 105211.

(59) Lee, H.; Lee, H.; Park, J. Y. Direct Imaging of Surface Plasmon-Driven Hot Electron Flux on the Au Nanoprism/TiO2. Nano Lett. 2019, 19 (2), 891-896.

(60) Cui, Q.; Yashchenok, A.; Li, L.; Möhwald, H.; Bargheer, M. Mechanistic Study on Reduction Reaction of Nitro Compounds Catalyzed by Gold Nanoparticles Using in Situ SERS Monitoring. Colloid Surf. A-Physicochem. Eng. Asp. 2015, 470, 108-113. 
(61) Boerigter, C.; Campana, R.; Morabito, M.; Linic, S. Evidence and Implications of Direct Charge Excitation as the Dominant Mechanism in Plasmon-Mediated Photocatalysis. Nat. Commun. 2016, 7 (1), 1-9.

(62) Tesema, T. E.; Kafle, B.; Tadesse, M. G.; Habteyes, T. G. Plasmon-Enhanced Resonant Excitation and Demethylation of Methylene Blue. J. Phys. Chem. C 2017, 121 (13), 7421-7428. 